\title{
The trinucleotide repeat expansion on chromosome 6p (SCA1) in autosomal dominant cerebellar ataxias
}

\author{
P. Giunti, ${ }^{1}$ M. G. Sweeney, ${ }^{1}$ M. Spadaro, ${ }^{2}$ C. Jodice,${ }^{3}$ A. Novelletto, ${ }^{3}$ P. Malaspina, ${ }^{3}$ M. Frontali ${ }^{4}$ and \\ A. E. Harding ${ }^{1}$
}

'University Department of Clinical Neurology, Institute of Neurology, London, UK, ${ }^{2}$ Istituto di Clinica delle Mallattie nervose e Mentali, University 'La Sapienza', '3 Dipartimento di Biologia, University 'Tor Vergata' and ${ }^{4}$ Istituto di Medicina Sperimentale, Rome, Italy

\author{
Correspondence to: Professor A. E. Harding, Institute of \\ Neurology, Queen Square, London WCIN 3BG, UK
}

\begin{abstract}
Summary
Affected members of 73 families with a variety of autosomal dominant late onset cerebellar ataxias (ADCAs) were investigated for the trinucleotide $(C A G)$ repeat expansion which is found in pedigrees exhibiting linkage to the SCAI locus on chromosome 6. Most of the families were too small for linkage analysis. The mutation was only found in ADCA type I, in 19 out of 38 such kindreds investigated (50\%). It was slightly more common in Italian (59\%) than British (50\%) families, and was also found in Malaysian, Bangladeshi and Jamaican kindreds. Overall, ADCA type I patients with the
\end{abstract}

Key words: dominant ataxia; trinucleotide repeat expansion had a lower incidence of hyporeflexia and facial fasciculation than those without. The trinucleotide expansion was not found in eight families with ADCA and maculopathy or 24 kindreds with a pure type of $A D C A$, confirming that these syndromes are genetically distinct. It was also not detected in 12 patients with sporadic degenerative ataxias. DNA analysis for the SCAI mutation is useful diagnostically in single patients or small families, and can be used for presymptomatic testing where appropriate.

\section{Introduction}

The autosomal dominant late onset cerebellar ataxias (ADCAs) are clinically and genetically heterogeneous. Family studies suggest that, compared with other syndromes which are genetically distinct, there is little clinical evidence of heterogeneity in families with ADCA associated with variable combinations of supranuclear ophthalmoplegia, optic atrophy, mild dementia, peripheral neuropathy or extrapyramidal dysfunction (Harding, 1982). There is, nevertheless, evidence for locus heterogeneity within this broad group (ADCA type I). Linkage studies have shown that the disease gene maps to the short arm of chromosome 6 in several large families (Zoghbi et al., 1988, 1993; Jodice et al., 1993; Kwiatkowski et al., 1993), and there is a locus on chromosome 12 in Cuban pedigrees (Gispert et al., 1993). It is difficult to distinguish these clinically, and the same applies to families with Machado-Joseph disease, a syndrome initially reported in individuals of Azorean/Portuguese descent (Coutinho and Andrade, 1978). The gene locus for Machado-Joseph disease has been mapped to chromosome $14 \mathrm{q}$ in both Japanese and
Portuguese families (Takiyami et al., 1993). However, an ADCA gene has also been mapped to this locus in three French families not considered to have Machado-Joseph disease on clinical grounds (Stevanin et al., 1994).

Autosomal dominant late onset cerebellar ataxia and pigmentary macular dystrophy (ADCA II) is clinically and genetically distinct from ADCA type I (Harding, 1982; Enevoldson et al., 1994). No positive linkage data have been reported in this disorder, although the disease locus is not linked to the HLA locus on chromosome 6p (Kumar et al., 1986). The pathological findings in ADCA types I and II are usually those of olivopontocerebellar atrophy, but nearly always with abnormalities elsewhere, including the basal ganglia, spinal cord and peripheral nerves. There is also a later onset (usually over 50 years) relatively 'pure' cerebellar syndrome in which ocular or extrapyramidal features do not occur (ADCA type III), with autopsy findings of cerebellar cortical atrophy with loss of cells in the dentate and/or olivary nuclei (Hoffman et al., 1971). No linkage studies have been 
Table 1 Clinical classification and results of SCAI mutation analysis

\begin{tabular}{lllll}
\hline Disease category & \multicolumn{3}{l}{ Number of families with mutation/total number of families (\%) } \\
\cline { 2 - 5 } & British & Italian & Other origins & Total \\
\hline ADCA I & $6 / 12(50)$ & $10 / 17(59)$ & $3 / 9^{\prime}(33)$ & $19 / 38(50)$ \\
ADCA II & $0 / 6$ & $0 / 1$ & $0 / 1$ & $0 / 8$ \\
ADCA III & $0 / 21$ & $0 / 1$ & $0 / 2$ & $0 / 24$ \\
Other dominant ataxias & & & & \\
With dementia/myoclonus & $0 / 1$ & - & - & $0 / 1$ \\
$\begin{array}{l}\text { With severe dementia } \\
\text { Intermittent ataxia }\end{array}$ & $0 / 1$ & - & - & $0 / 1$ \\
'Idiopathic' late onset & $0 / 1$ & - & - & $0 / 1$ \\
$\quad$ cerebellar ataxia (cases) & $0 / 9$ & $0 / 1$ & & $0 / 12$ \\
\hline
\end{tabular}

reported in this disorder, apart from exclusion of linkage to $6 p$ in a family with rather earlier onset and a fairly indolent course (Frontali et al., 1992).

Recently, the mutation in the ADCA gene on chromosome $6 \mathrm{p}$ (at the locus termed SCA1) has been identified (Chung et al., 1993; Orr et al., 1993). It is an unstable expanded trinucleotide $(\mathrm{CAG})$ repeat sequence, with similar characteristics to the Huntington's disease mutation (Huntington's Disease Collaborative Group 1993). There is an inverse correlation between repeat length and age of onset of the disease. This discovery makes it possible to determine if affected members of families too small for linkage analysis have the SCA1 mutation. The results of such a study in 73 families with various forms of ADCA and 12 patients with late onset degenerative ataxias who had no history of affected relatives are reported in this paper.

\section{Patients and methods \\ Patients}

DNA was analysed from at least one member of 73 families with a variety of dominant ataxias (Table 1). Families were considered to have ADCA type I if the age of onset was $<50$ years in the majority of affected family members, and at least one patient per family had features such as supranuclear ophthalmoplegia, optic atrophy, peripheral neuropathy, dementia and fasciculations of the limbs and/or face during their illness. Also included in this group is one family where the only patient examined had a pure cerebellar syndrome at an early stage of the disease ( $<5$ years duration), as is common in ADCA type I (Harding, 1982). Inclusion in this category was warranted by consistently early onset in the family ( $<45$ years) and the presence of cerebellar and brainstem atrophy seen on imaging studies, suggesting a pathological substrate of olivopontocerebellar atrophy rather than cerebello-olivary atrophy which is usually seen in $\mathrm{ADCA}$ type III. Three of the 12 British families with ADCA type I were in the study of Harding (1982), including families 1 and 2; one of these (family 2) was known to have a locus on chromosome 6 on the basis of linkage analysis (Shrimpton et al., 1993). Of the 17 Italian families with ADCA type I, seven were known to have a locus on chromosome $6 p$ (Conighi et al., 1984; Spadaro et al., 1992; Jodice et al.. 1993), and in two this had been excluded (unpublished data).

Type II ADCA was defined by the consistent finding of maculopathy in affected family members (Harding, 1982: Enevoldson et al., 1994). Type III ADCA was defined as a pure cerebellar syndrome, occasionally with distal loss of vibration sense or pyramidal signs in the legs, but without the additional features seen in ADCA type I referred to above, after disease durations $>5$ years in all affected family members. This syndrome is probably heterogeneous. In 11 families, the age of onset was consistently late, over 40 in all patients and 50 or over in all but three. In the other 13 (one Italian; Frontali et al., 1992), onset was earlier, ranging between the second to fourth decade of life. In all these 13 families the disease course was only slowly progressive, with patients not losing the ability to walk until 30 or 40 years after onset. Three families with rare or less well characterized dominant ataxias were also studied, of which details are given in Table 1.

Fifty-six of the families were studied clinically in the UK by P.G. or A.E.H. They originated from the UK (42), India, Pakistan or Bangladesh (three), the West Indies (three), Italy (two), Malaysia (one), the Philippines (one), Brazil (one), China (one) Denmark (one) and Belgium (one). The 17 families studied in Italy (all Italian) were assessed clinically by M.S. or P.G. We also investigated 12 patients (11 in the UK, one in Italy) with 'idiopathic' late onset cerebellar ataxia (Harding, 1981), in whom there was no history of affected relatives. Nine of these were British, one Afro-Caribbean, one Indian and one Italian.

For clinical comparisons, age of onset data were compared using the Mann-Whitney test, and the presence or absence of clinical features using $\chi^{2}$ analysis of raw data with Yates' correction.

\section{DNA analysis}

In London, analysis of the trinucleotide repeat in the SCAI gene was performed in UK-based patients and 44 healthy 
Table 2 Comparison of clinical features of ADCA I families with and without the SCA I mutation

\begin{tabular}{lll}
\hline & SCA1 & Not SCA1 \\
\hline $\begin{array}{l}\text { Median age at onset (years) in } \\
\text { all patients (range) }\end{array}$ & $\begin{array}{l}34(17-53) \\
(n=103)\end{array}$ & $\begin{array}{l}34(9-54) \\
(n=69)\end{array}$ \\
& Percentage of cases* & \\
Supranuclear ophthalmoplegia & 63 & 67 \\
Staring gaze & 5 & 10 \\
Dysphagia & 41 & 46 \\
Cognitive impairment & 8 & 10 \\
Extrapyramidal features & 14 & 4 \\
Fasciculation & 21 & $48\left(\chi^{2}=4.5, P=0.03\right)$ \\
$\quad$ Face/tongue & 20 & 24 \\
Limbs & 67 & 31 \\
Increased reflexes & 15 & 42 \\
Depressed/absent reflexes & 37 & 36 \\
Sensory loss & & \\
\hline
\end{tabular}

*From available clinical data on 46 SCAI patients and 30 non-SCAl patients.

control subjects, using a modification of the method described (Orr et al., 1993). The polymerase chain reaction (PCR) was carried out in a $25 \mu \mathrm{l}$ reaction volume using $100 \mathrm{ng}$ DNA, $1.5 \mathrm{mM} \mathrm{MgCl}_{2}, 50 \mathrm{mM} \mathrm{KCl}, 10 \mathrm{mM}$ Tris, pH 8. $100 \mu \mathrm{M}$ dATP, $10 \mu \mathrm{M}$ dCTP, $100 \mu \mathrm{M}$ dTTP, $100 \mu \mathrm{M}$ dGTP, 10 pmol each primer, $0.4 \mu \mathrm{Ci}\left[\alpha-{ }^{32}\right] \mathrm{PdCTP}$ and 2.5 units Taq polymerase. Primers were as described (Orr et al., 1993). Initial DNA denaturation at $94^{\circ} \mathrm{C}$ for $3 \mathrm{~min}$ was followed by 25 cycles of $30 \mathrm{~s}$ each at 94,57 and $72^{\circ} \mathrm{C}$, respectively, and a final elongation step of $10 \mathrm{~min}$ at $72^{\circ} \mathrm{C}$. Products of the PCR were fractionated on $6 \%$ denaturing acrylamide gels. Allele sizes were estimated using an M13 sequencing ladder, which we consider gives an accuracy of +2 repeat units; the SCAI mutation was defined by the presence of $>42$ repeats in one allele (Orr et al., 1993). The patients based in Italy were investigated in Rome using the method reported by Orr et al. (1993).

\section{Results}

The classification of the families is shown in Table 1. as is the number of kindreds with the SCAl mutation. Ten of 17 families originating from Italy with ADCA type I (59\%) had the mutation, compared with six of 12 families of British origin $(50 \%)$, and three of nine other kindreds. The last were Malaysian, Jamaican and Bangladeshi; other ADCA type I families from India, China, the West Indies, Brazil, Belgium and Denmark did not have the SCAI mutation. Not surprisingly, all of the eight families who had shown linkage to chromosome 6p markers had the SCAl expansion and this was not present in the two ADCA type I families in which linkage to this region had been excluded. The characteristics of the SCA1 mutation in these families have been reported elsewhere (Jodice et al., 1994), confirming the relationship between repeat length, age of onset and severity and more marked instability during paternal transmission (Orr et al., 1993).

A comparison of the clinical features of ADCA type I in patients with and without the SCAl expansion is given in Table 2. The median and ranges of age at onset of symptoms were very similar. Overall, the non-SCAl patients had a higher incidence of depressed or absent, as opposed to increased, reflexes. Reflex loss increases with disease duration in ADCA type I (Harding, 1982), but there was a higher proportion of non-SCAI cases with hyporeflexia (four out of 11 compared with three out of 31 SCA1, $\chi^{2}=10.7, P=$ 0.004 ) in patients with disease durations of 7 years or less. In longer duration cases there was no significant difference between the two groups in terms of incidence of hyporeflexia (four out of $15 \mathrm{SCA} 1$, seven out of 17 non-SCA1). The other significant finding was that more non-SCAl patients had facial or lingual fasciculation. This was not related to disease duration (data not shown).

None of the seven families with ADCA type II (ataxia and macular dystrophy), or the 24 with the 'pure' ADCA type III, had the SCAI mutation, and the same applied to those with less common dominant disorders, including a family with intermittent ataxia, and the 12 patients without affected relatives.

\section{Discussion}

This study confirms the previous clinical observations which separated the pure type of ADCA (type III) and that with maculopathy (type II) from the more common and variable multisystem syndrome ADCA type I (Harding, 1982). The SCAl expansion was only observed in patients with ADCA type $\mathrm{I}$, in half of the families originating from the UK and slightly more of those of Italian origin. This may reflect a degree of ascertainment bias in the Italian families as these were collected initially for chromosome 6 linkage studies, 
and certainly one was known to be 6p-linked in 1984 (Conighi et al., 1984). Also, four of the SCA1 families are from Calabria and probably reflect a founder effect in this part of Italy. The British families were ascertained solely by systematic DNA storage from all families with ADCA seen by A.E.H. over a 7 year period, and a more recent intensive collection of British families with any type of dominant ataxia. In the French population, six of the 10 families for whom linkage analysis information is available showed evidence for a locus on chromosome 6 (Khati et al., 1993), a similar proportion to that observed in our European patients. The presence of the SCA1 mutation in Malaysian, Jamaican and Bangladeshi kindreds suggests that this genetic defect is not confined to Caucasian populations, and may well have multiple origins in different racial groups, as is the case in Huntington's disease. SCA1 has previously been described in Black American and Cape coloured (South African) families (Zoghbi et al., 1988; Bryer et al., 1992). However, SCAl as defined by linkage analysis has been reported most commonly in families of European origin (Zoghbi et al., 1993), and other types of ADCA may be more frequent in certain populations. This clearly applies to Azorean or Portuguese families, and possibly the Japanese (Takiyama et al., 1993; Zoghbi et al., 1993).

As in the French study (Khati et al., 1993), in which 6plinked $\mathrm{ADCA}$ was indistinguishable from non-6p-linked ADCA, we could identify relatively few clinical differences between ADCA type I patients with the SCAI mutation and those without. The latter tend to exhibit depressed or absent tendon reflexes, as opposed to hyperreflexia, in the first 7 years of the disease. The higher incidence of facial fasciculation in the non-SCA1 families is of interest as this has been reported frequently in patients with Machado-Joseph disease (Coutinho and Andrade, 1978) and in the Cuban families with a disease locus on chromosome 12 (Orozco Diaz et al., 1990). It should be stressed that these were group differences derived from a relatively large number of patients and do not serve to distinguish SCA1 from other types of ADCA type $I$ in individual patients or families. Our non-SCAI families are likely to be genetically heterogeneous and presumably have disease genes on chromosomes 12,14 or elsewhere (Stevanin et al., 1994). It seems highly probable that all the mutations underlying ADCA type I, and ADCA type II which exhibits striking anticipation and a paternal effect (Enevoldson et al., 1994), will be unstable trinucleotide repeats.

Most of our families with the expansion were too small for linkage analysis, and direct mutation detection is clearly very useful in clinical practice in making a diagnosis of SCAl in such kindreds. If applicable, this analysis can be used for presymptomatic diagnosis, and we have already performed this in two at risk subjects, using the same preand post-test counselling protocols as in Huntington's disease families (World Federation of Neurology Research Group on Huntington's disease, 1989).

\section{Acknowledgements}

We wish to thank many colleagues for referring families and helping in obtaining blood samples or clinical information. The work was supported by the Friedreich's Ataxia Group (UK), the Glyn Worsnip/Raymond Barnes ataxia fund, CNR PF Ingegneria Genetica and Telethon Italia (grant no. 358).

\section{References}

Bryer A, Martell RW, du Toit ED, Beighton P. Adult onset spinocerebellar ataxia linked to HLA in a South African kindred of mixed ancestry. Tissue Antigens 1992: 40: 111-15.

Chung M, Ranum LPW, Duvick LA, Servadio A, Zoghbi HY, On HT. Evidence for a mechanism predisposing to intergenerational CAG repeat instability in spinocerebellar ataxia type I. Nature Genet 1993: 5: 254-8.

Conighi C, Granieri E, Tola R, Sensi A, Mattiuz PL. HLA and spinocerebellar ataxia: study of a family. Ital J Neurol Sci 1984: 5 Suppl 2: 154-8.

Coutinho P, Andrade C. Autosomal dominant system degeneration in Portuguese families of the Azores islands. Neurology 1978: 28: 703-9.

Enevoldson PG, Sanders MD. Harding AE. Autosomal dominant cerebellar ataxia with macular dystrophy: a clinical and genetic study of eight families. Brain 1994; 117: 445-60.

Frontali M, Spadaro M, Giunti P, Bianco F, Jodice C, Persichetti F. et al. Autosomal dominant pure cerebellar ataxia. Neurological and genetic study. Brain 1992; 115: 1647-54.

Gispert S, Twells R, Orozco G, Brice A, Weber J, Heredero L. et al. Chromosomal assignment of the second locus for autosomal dominant cerebellar ataxia (SCA2) to chromosome 12q23-24.1. Nature Genet 1993; 4: 295-9.

Harding AE. 'Idiopathic' late onset cerebellar ataxia. A clinical and genetic study of 36 cases. J Neurol Sci 1981; 51: 259-71.

Harding AE. The clinical features and classification of the late onset autosomal dominant cerebellar ataxias: a study of 11 families. including descendants of the 'Drew family of Walworth'. Brain 1982; 105: 1-28.

Hoffman PM, Stuart WH, Earle KM, Brody JA. Hereditary lateonset cerebellar degeneration. Neurology 1971; 21: 771-7.

Huntington's Disease Collaborative Group. A novel gene containing a trinucleotide repeat that is expanded and unstable on Huntington's disease chromosomes. Cell 1993; 72: 971-83.

Jodice C, Frontali M, Persichetti F, Novelletto A, Pandolfo M, Spadaro M, et al. The gene for spinal cerebellar ataxia 1 (SCA1) is flanked by two closely linked highly polymorphic microsatellite loci. Hum Mol Genet 1993; 2: 1383-7.

Jodice C, Malaspina P, Persischetti F, Novelletto A, Spadaro M, Giunti P, et al. Effect of trinucleotide repeat length and parental sex on phenotypic variation in spinocerebellar ataxia 1. Am J Hum Genet. 1994; 54: 959-65.

Khati C, Stevanin G, Durr A, Chneiweiss H, Belal S, Seck A et al. 
Genetic heterogeneity of autosomal dominant cerebellar ataxia type 1: clinical and genetic analysis of 10 French families. Neurology 1993; $43: 1131-7$.

Kumar D, Blank CE, Gelsthorpe K. Hereditary cerebellar ataxia and genetic linkage with HLA. Hum Genet 1986; 72: 327-32.

Kwiatkowski TJ, Orr HT, Banfi S, McCall AE, Jodice C, Persichetti $\mathrm{F}$, et al. The gene for autosomal dominant spinocerebellar ataxia (SCA1) maps centromeric to D6S89 and shows no recombination, in nine large kindreds, with a dinucleotide repeat at the AMIO locus. Am J Hum Genet 1993; 53: 391-400.

Orozco Diaz G, Nodarse Fleites A, Cordoves Sagaz R, Auburger G. Autosomal dominant cerebellar ataxia: clinical analysis of 263 patients from a homogeneous population in Holguin, Cuba. Neurology 1990; 40: 1369-75.

Orr HT, Chung MY, Banfi S, Kwiatkowski TJ, Servadio A, Beaudet $A$, et al. Expansion of an unstable trinucleotide CAG repeat in spinocerebellar ataxia type 1. Nature Genet 1993; 4: 221-6.

Shrimpton AE, Davidson R, MacDonald N, Brock DJH. Presymptomatic testing for autosomal dominant spinocerebellar ataxia type I. J Med Genet 1993; 30: 616-17.

Spadaro M, Giunti P, Lulli P, Frontali M, Jodice C, Cappellacci S, et al. HLA-linked spinocerebellar ataxia: a clinical and genetic study of large Italian kindreds. Acta Neurol Scand 1992; 85: 257-65.
Stevánin G, Le Guern E, Ravisé N, Chneiweiss H, Dürr A, Cancel $\mathrm{G}$, et al. A third locus for autosomal dominant cerebellar ataxia type I maps to chromosome 14q24.3-qter: evidence for the existence of a fourth locus. Am J Hum Genet 1994; 54: 11-20.

Takiyama Y, Nishizawa M, Tanaka H, Kawashima S, Sakamoto H, Karube $\mathrm{Y}$, et al. The gene for Machado-Joseph disease maps to human chromosome 14q. Nature Genet 1993; 4: 300-4.

World Federation of Neurology: Research Committee. Research Group on Huntington's disease. Ethical issues policy statement on Huntington's disease molecular genetics predictive tests. J Neurol Sci $1989 ; 94: 327-32$.

Zoghbi HY, Pollack MS, Lyons LA, Ferrell RE, Daiger SP, Beaudet AL. Spinocerebellar ataxia: variable age of onset and linkage to human leukocyte antigen in a large kindred. Ann Neurol 1988; 23; $580-4$.

Zoghbi HY, Frontali M, Orr HT, Sandkuijl L, Cann H, Sasaki H. et al. Linkage studies in dominantly inherited ataxias. Adv Neurol 1993; 61: 133-7.

Received December 31, 1993. Revised February 23, 1994. Accepted March 18, 1994 